\title{
CheckMate 153 study: are age and performance status relevant for immune checkpoint inhibitor efficacy?
}

\author{
Jose Carlos Benitez ${ }^{1}$,Jordi Remon ${ }^{2}$, Benjamin Besse ${ }^{1,3}$ \\ ${ }^{1}$ Gustave Roussy, Department of Cancer Medicine, Villejuif, France; ${ }^{2}$ Department of Medical Oncology, Centro Integral Oncológico Clara Campal \\ (HM-CIOCC), Hospital HM Delfos, HM Hospitales, Barcelona, Spain; ${ }^{3}$ Paris-Sud University Bicetre, Orsay, France \\ Correspondence to: Benjamin Besse, MD, PhD. University Paris-Sud and Gustave Roussy Cancer Campus, 114 Rue Edouard Vaillant, 94805 Villejuif, \\ France. Email: Benjamin.BESSE@gustaveroussy.fr. \\ Provenance: This is an invited article commissioned by the Academic Editor Hexiao Tang, MD, PhD (Division of Thoracic Surgery, Massachusetts \\ General Hospital, Harvard Medical School, Boston, MA, USA; Division of Thoracic Surgery, Zhongnan Hospital of Wuhan University, Wuhan, \\ China). \\ Comment on: Spigel DR, McCleod M, Jotte RM, et al. Safety, Efficacy, and Patient-Reported Health-Related Quality of Life and Symptom Burden \\ with Nivolumab in Patients with Advanced Non-Small Cell Lung Cancer, Including Patients Aged 70 Years or Older or with Poor Performance \\ Status (CheckMate 153). J Thorac Oncol 2019;14:1628-39.
}

Submitted Dec 01, 2019. Accepted for publication Dec 19, 2019.

doi: $10.21037 /$ tlcr.2019.12.26

View this article at: http://dx.doi.org/10.21037/tlcr.2019.12.26

Immune checkpoint inhibitors (ICIs), including pembrolizumab and nivolumab, both monoclonal antibodies against programmed cell death-1 (PD-1), and antibodies targeting the programmed cell death receptor ligand-1 (PD-L1) such as atezolizumab or durvalumab, have heralded impressive therapeutic advances in previously treated advanced nonsmall cell lung cancer (NSCLC). Several studies have compared ICI with standard chemotherapy, and reported an unprecedented 5 -year overall survival (OS) of $15 \%$ with ICIs (1). This breakthrough was followed by a further report of a survival benefit when ICIs were administered in the firstline setting in an unselected population, both as single agents and in combination with chemotherapy or ipilimumab, compared with platinum-based chemotherapy (2).

Nonetheless, due to concerns regarding the risk-benefit ratio, patients with an ECOG performance status (PS) $\geq 2$ are excluded or underrepresented in the contributing phase III clinical trials, despite the fact that they represent up to $25 \%$ of newly diagnosed or recurrent patients with NSCLC (3). Similarly, although median age at diagnosis is over 70 in almost $50 \%$ of cases, with $15 \%$ of the population being more than 85 years (4), this older population is also not represented in clinical trials. Additionally, $50 \%$ of elderly patients in daily clinical practice have an ECOG PS of 2 (5). Two key points arising from this bias in "over-selecting" the eligible population for phase III trials evaluating ICIs are firstly that it may explain the outcome discrepancies with the real-world population treated with ICIs (6), and secondly, the efficacy of ICIs in the elderly population and in patients with ECOG PS 2 (elderly or not) is unknown.

Various clinical trials in pre-treated NSCLC patients, such as the CheckMate 171 (7), CheckMate 169 (8), TAIL (9) and $\mathrm{PeP} 2$ trials $(10)$, as well as pooled analyses $(11,12)$ and retrospective evaluations $(5,13-16)$ have shed light on the outcomes with ICIs in these frail populations. The phase IIIB/IV CheckMate 153 study reported by Spigel et al. (17) describes as a primary endpoint the safety [incidence of grade 3 to 5 selected treatment-related adverse events (TRAEs)] and outcome of nivolumab in 1,426 advanced unselected previously treated NSCLC patients. Importantly, the subgroups of frail patients were large, and included both elderly patients ( $\geq 70$ years, $\mathrm{N}=556,39 \%$ ) and patients with ECOG PS $\geq 2$ (N=128, 9\%). Of note, PD-L1 expression $<1 \%$ and $\geq 50 \%$ was reported in the same proportion in the overall population as well as in both subgroups, reaching $40 \%$ and $20 \%$, respectively. Similar incidence of selected grade 3 to 5 TRAEs (6-9\%) and grade 3 or 4 TRAEs $(12-14 \%)$ were reported between subgroups and the overall population. The median OS in the overall population was of 9.1 and 10.3 months in patients aged 
$\geq 70$ years. Patients with an ECOG PS of 2 or more presented a shorter median OS (4.0 months). In the global population, OS was longer in PD-L1 positive tumours, however, OS according to PD-L1 expression in the frail populations is not reported. The most common reason for treatment discontinuation was disease progression, with a $50 \%$ progression rate in the overall population and in both subgroups.

These CheckMate 153 survival and safety data mirror those reported in a pooled analysis of pivotal phase III clinical trials with nivolumab (CheckMate 017 and CheckMate 057) (18), however in the latter trials, the proportion of patients aged $\geq 75$ years was below $10 \%$ and ECOG PS $\geq 2$ patients were excluded. In contrast with the overall population, nivolumab was not associated with an increased OS benefit in 72 elderly patients ( $\geq 75$ years) compared with chemotherapy $(\mathrm{HR}=1.19)(1)$. This is of relevance as some studies have reported that elderly patients ( $\geq 70$ years) had shorter PFS and OS than younger individuals, without a difference in immune related adverse events, but without reported stratification according to ECOG PS (19).

Real-world studies in elderly patients (defined as age $\geq 75$ years) have demonstrated no differences in clinical outcomes with nivolumab compared to non-elderly patients, whereas those with a poor ECOG PS $(\geq 2)$ had inferior outcomes even when adjusting for age (20). Other realworld cohorts $(5,6,13)$ have reported that the benefit with ICIs in previously-treated and elderly NSCLC patients was comparable to younger counterparts, even using different age cut-offs, and some retrospective data have reported efficacy of patients aged $\geq 80$ years, albeit with small sample sizes (5,21). Similarly, among 10,452 French NSCLC patients who initiated nivolumab in 2015 as second-line therapy or beyond, $514(4.9 \%)$ were 80 years or over (median age 82.5 years), and their median OS was similar to non-elderly patients (11.5 months in both age-subgroups). In this cohort, comorbidities were statistically less frequent in the elderly group $(\mathrm{P}<0.001)$, which may reflect an overselection even in the routine setting (22). Octogenarians may get benefit from this ICI, but comorbidities and PS are relevant for making treatment decisions in this subgroup. Importantly, the upper age limit for ICIs, if of value, has not been established. Data coming from a recent meta-analysis enrolling 5,265 cancer patients from nine randomized controlled trials did not observed differential efficacy of ICIs according to age. However, this meta-analysis only included two trials concerning NSCLC. Sixteen percent of all patients, 854 of 5,265 patients, were enrolled. The exploratory subgroup analysis did not report significant OS benefit with anti-PD-1 agents in patients older than 75 years (12). Although the CheckMate 153 trial (17) enrolled patients $\geq 70$ years, the proportion of patients aged $\geq 75$ or $\geq 80$ remains unknown so firm conclusions in these specific subgroups of age cannot be made.

One concern is the potential correlation between the elderly and an immune phenotype of primary resistance through a paradoxically higher concentration of inflammatory cytokines and autoantibodies, a phenomenon probably linked to the progressive and continuous deterioration of the immune system functions with ageing, known as immunosenescence $(23,24)$. In cancer patients, older age ( $\geq 65$ years) during ICI treatment has been correlated with increased risk of hyper-progressive disease (25), however, this association was not observed in a cohort of NSCLC patients (26) or in the CheckMate 153 study, with a $50 \%$ progression rate in the overall population and both subgroups (17). Indeed, immunosenescence defined by a CD28-CD $57^{+} \mathrm{KLRG} 1^{+}$phenotype on peripheral T-lymphocytes, which occurs in one-third of advanced NSCLC patients and correlates with a lower disease control rate for ICIs, is independent of age (27).

Results of the CheckMate 153 trial in ECOG PS $\geq 2$ patients suggest that safety with ICIs is consistent with the overall population, although it is known that tolerance of chemotherapy is worse (17). However, efficacy is limited with a median OS ranging from 3.4 to 5.9 months (5-17), suggesting poor PS is a negative predictive and prognostic factor for ICI treatment. Surprisingly, the PeP2 study assessing the role of pembrolizumab in 60 patients with $\mathrm{ECOG} P S \geq 2$ reported a response rate of $25.5 \%$ and median progression-free survival and OS of 6.0 and 12.1 months, respectively, with $12 \%$ grade $\geq 3$ adverse events. Different factors contribute to patients' PS scoring such as age, symptoms related to lung cancer and comorbidities. Therefore, discrepancies in any of these characteristics in the $\mathrm{PeP} 2$ study for selecting PS 2 patients may have contributed to explain differences in outcome. The predictive role of PD-L1 expression seems controversial in ECOG PS $\geq 2$ patients, as despite that $20 \%$ of ECOG PS $\geq 2$ patients in CheckMate 153 (17) and PeP2 (10) having tumors expressing PD-L1 $\geq 50 \%$, median OS is three times longer in the PeP2 trial (10).

Clearly, besides chronological age, an optimal geriatric assessment, along with validated fragility and comorbidity scales, such as FRAGIL, polypharmacy or the Charlson 
index, may be necessary to obtain a global medical picture with the aim to select elderly patients and ECOG PS $\geq 2$ patients who may obtain most benefit from ICI therapy.

The CheckMate 153 study endorses ICI efficacy in previously-treated elderly patients and suggests ICIs as an alternative treatment strategy in ECOG PS $\geq 2$ patients with their better safety profile than chemotherapy. Stratifying the benefit according to geriatric assessment and PS in elderly patients and defining the optimal ECOG PS $\geq 2$ patients for receiving ICIs, based on age, comorbidities and diseaserelated factors, are future achievable challenges for defining the optimal ICI therapy in these subgroups.

\section{Acknowledgments}

None.

\section{Footnote}

Conflicts of Interest: The authors have no conflicts of interest to declare.

Ethical Statement: The authors are accountable for all aspects of the work in ensuring that questions related to the accuracy or integrity of any part of the work are appropriately investigated and resolved.

\section{References}

1. Gettinger S, Borghaei H, Brahmer J, et al. FiveYear Outcomes From the Randomized, Phase 3 Trials CheckMate 017/057: Nivolumab vs Docetaxel in Previously Treated NSCLC. J Thorac Oncol 2019;14:S244-5.

2. Remon J, Ahn M-J, Girard N, et al. Advanced-Stage NonSmall Cell Lung Cancer: Advances in Thoracic Oncology 2018. J Thorac Oncol 2019;14:1134-55.

3. Salloum RG, Smith TJ, Jensen GA, et al. Using claimsbased measures to predict performance status score in patients with lung cancer. Cancer 2011;117:1038-48.

4. Available online: https://seer.cancer.gov/statfacts/html/ lungb.html

5. Galli G, De Toma A, Pagani F, et al. Efficacy and safety of immunotherapy in elderly patients with non-small cell lung cancer. Lung Cancer 2019;137:38-42.

6. Khozin S, Carson KR, Zhi J, et al. Real-World Outcomes of Patients with Metastatic Non-Small Cell Lung Cancer Treated with Programmed Cell Death Protein 1 Inhibitors in the Year Following U.S. Regulatory Approval. Oncologist 2019;24:648-56.

7. Popat S, Ardizzoni A, Ciuleanu T, et al. Nivolumab in previously treated patients with metastatic squamous NSCLC: Results of a European single-arm, phase 2 trial (CheckMate 171) including patients aged $\geq 70$ years and with poor performance status. Ann Oncol 2017;28:v460-96.

8. Juergens R, Chu Q, Rothenstein J, et al. P2.07-029 CheckMate 169: Safety/Efficacy of Nivolumab in Canadian Pretreated Advanced NSCLC (including Elderly and PS 2) Patients. J Thorac Oncol 2017;12:S2426-27.

9. Ardizzoni A, Azevedo S, Rubio Viquiera B, et al. Primary results from TAIL, a global single-arm safety study of atezolizumab (atezo) monotherapy in a diverse population of patients with previously treated advanced non-small cell lung cancer (NSCLC). Ann Oncol 2019;30:mdz394.082.

10. Middleton G, Brock K, Summers Y, et al. Pembrolizumab in performance status 2 patients with non-small cell lung cancer (NSCLC): Results of the PePS2 trial. Ann Oncol 2018;29:mdy292.007.

11. Landre T, Taleb C, Nicolas $\mathrm{P}$, et al. Is there a clinical benefit of anti-PD-1 in patients older than 75 years with previously treated solid tumour? J Clin Oncol 2016;34:abstr 3070.

12. Nishijima TF, Muss HB, Shachar SS, et al. Comparison of efficacy of immune checkpoint inhibitors (ICIs) between younger and older patients: A systematic review and metaanalysis. Cancer Treat Rev 2016;45:30-7.

13. Grossi F, Genova C, Crinò L, et al. Real-life results from the overall population and key subgroups within the Italian cohort of nivolumab expanded access program in non-squamous non-small cell lung cancer. Eur J Cancer 2019;123:72-80.

14. Katsura H, Suga Y, Araya T, et al. Efficacy and Safety of Nivolumab in Patients with Advanced Non-small-cell Lung Cancer and Poor Performance Status. J Cancer 2019;10:2139-44.

15. Ksienski D, Wai ES, Croteau N, et al. Pembrolizumab for advanced nonsmall cell lung cancer: Efficacy and safety in everyday clinical practice. Lung Cancer 2019;133:110-6.

16. Molinier O, Audigier-Valette C, Cadranel J, et al. OA 17.05 IFCT-1502 CLINIVO: Real-Life Experience with Nivolumab in 600 Patients (Pts) with Advanced NonSmall Cell Lung Cancer (NSCLC). J Thorac Oncol 2017;12:S1793.

17. Spigel DR, McCleod M, Jotte RM, et al. Safety, Efficacy, and Patient-Reported Health-Related Quality of Life 
and Symptom Burden with Nivolumab in Patients with Advanced Non-Small Cell Lung Cancer, Including Patients Aged 70 Years or Older or with Poor Performance Status (CheckMate 153). J Thorac Oncol 2019;14:1628-39.

18. Horn L, Spigel DR, Vokes EE, et al. Nivolumab Versus Docetaxel in Previously Treated Patients With Advanced Non-Small-Cell Lung Cancer: Two-Year Outcomes From Two Randomized, Open-Label, Phase III Trials (CheckMate 017 and CheckMate 057). J Clin Oncol 2017;35:3924-33.

19. Corral de la Fuente E, Barquín García A, Saavedra Serrano C, et al. Benefit of immunotherapy (IT) in advanced nonsmall cell lung cancer (NSCLC) in elderly patients (EP). Ann Oncol 2019;30:mdz063.067.

20. Bagley S, Kothari S, Aggarwal C, et al. P3.02c-028 Outcomes of Nivolumab in Elderly Patients (pts) with Non-Small Cell Lung Cancer (NSCLC). J Thorac Oncol 2017;12:S1289.

21. Mebarki S, Gisselbrecht M, Fabre E, et al. Efficacy and safety of immune checkpoint inhibitors (ICIs) for treatment of advanced solid tumours in octogenarian patients. Ann Oncol 2019;30:mdz253.114.

22. Assié J, Cotté F, Levra MG, et al. P2.04-03 Nivolumab

Cite this article as: Benitez JC, Remon J, Besse B. CheckMate 153 study: are age and performance status relevant for immune checkpoint inhibitor efficacy? Transl Lung Cancer Res 2019;8(Suppl 4):S464-S467. doi: 10.21037/tlcr.2019.12.26
Outcomes in Octogenarian Patients with Advanced NonSmall Cell Lung Cancer in French Real-World Setting J Thorac Oncol 2019;14:S708.

23. Ferrara R, Mezquita L, Auclin E, et al. Immunosenescence and immunecheckpoint inhibitors in non-small cell lung cancer patients: does age really matter? Cancer Treat Rev 2017;60:60-8.

24. Daste A, Domblides C, Gross-Goupil M, et al. Immune checkpoint inhibitors and elderly people: A review. Eur J Cancer 2017;82:155-66.

25. Champiat S, Dercle L, Ammari S, et al. Hyperprogressive Disease Is a New Pattern of Progression in Cancer Patients Treated by Anti-PD-1/PD-L1. Clin Cancer Res 2017;23:1920-8.

26. Ferrara R, Caramella C, Texier M, et al. 1306PDHyperprogressive disease (HPD) is frequent in non-small cell lung cancer (NSCLC) patients (pts) treated with anti PD1/PD-L1 monoclonal antibodies (IO). Ann Oncol 2017;28:mdx380.009.

27. Ferrara R, Naigeon M, Auclin E, et al. P1.01-18 Immunosenescence Correlates with Progression upon PD-(L)-1 Blockade (IO) in Advanced Non-Small Cell Lung Cancer (aNSCLC) Patients. J Thorac Oncol 2018;13:S466-7. 